\title{
1 Retinal self-organization: a model of RGC and SAC mosaic formation
}

\section{Short title: Computational model of retinal mosaic formation}

3 Jean de Montigny ${ }^{1 *}$, Evelyne Sernagor ${ }^{1}$ and Roman Bauer ${ }^{2}$

\section{Affiliations}

$5 \quad{ }^{1}$ Biosciences Institute, Newcastle University, Newcastle upon Tyne, United Kingdom,

$6 \quad 2$ Department of Computer Science, University of Surrey, United Kingdom,

$7 *$ Corresponding author

8 E-mail: Jean.de-Montigny@newcastle.ac.uk

\section{Abstract}

10 Individual retinal cell types exhibit semi-regular spatial patterns called retinal mosaics. These

11 mosaics enable uniform sampling of visual information and are formed to varying degrees across

12 cell types. Retinal ganglion cells (RGC) and amacrine cells (including starburst amacrine cells

13 (SAC)) are notably known to exhibit such layouts. Mechanisms responsible for the formation of

14 such organised structures and their requirements are still not well understood. Mosaic formation

15 follows three main principles: (1) homotypic cells prevent nearby cells from adopting the same

16 type, (2) cell tangential migration, with homotypic cell repulsion, (3) cell death (with RGCs

17 exhibiting high rates of apoptosis).

18 Here, we use BioDynaMo, an agent-based simulation framework, to build a detailed and

19 mechanistic model of mosaic formation. In particular, we investigate the implications of the three

20 theories for RGC's mosaic formation. We report that the cell migration mechanism yields the most

21 regular mosaics and that cell death can create regular mosaics only if the death rate is kept below

$2230 \%$, after which cell death has a negative impact on mosaic regularity. In addition, and in

23 accordance with recent studies, we propose here that low density RGC type mosaics exhibit on 
24 average low regularities, and thus we question the relevance of regular spacing as a criterion for a

25 group of RGCs to form a RGC type.

26 We also investigate SAC mosaics formation and possible interactions between the ganglion cell

27 layer (GCL) and inner nuclear layer (INL) populations. Investigations are conducted both

28 experimentally and by applying our simulation model to the SAC population. We report that

29 homotypic interactions between the GCL and INL populations during mosaics creation are required

30 to reproduce the observed SAC mosaics' characteristics. This suggests that the GCL and INL

31 populations of SACs might not be independent during retinal development.

\section{Author Summary}

33 Retinal function depends on cells self-organisation during early development. Understanding the

34 mechanisms underlying this self-organisation could improve not only our comprehension of the

35 retina and its development but also of the cortex. Ultimately, this could lead to novel therapeutic approaches for developmental diseases. Computational models can be of precious help to study this process of self-organisation, given that they are biologically plausible. In this sense, it is important that implemented developmental mechanisms follow the principle of locally available information, without any global knowledge or external supervisor. Here, we follow this principle to investigate mosaic formation during retinal development. In this work, we demonstrate that tangential migration is the only mechanism able to form regular mosaics and that the GCL/INL SAC

42 populations might not be independent during their mosaic formation. More, we question the

43 relevance of regular spacing for RGC types classification.

\section{Introduction}

45 The mammalian retina is composed of six main types of neuronal cells (cones, rods, horizontal,

46 bipolar, amacrine and ganglion cells), subdivided into many different anatomical and functional

47 subtypes, forming a complexly organised structure. Notably, individual cell types exhibit semi- 
regular spatial patterns called retinal mosaics. Regular spacing between homotypic cells enable

49 homogeneous processing of the light signals, leaving no perceptual holes within our visual field. In

50 particular, sub-groups of retinal ganglion cells (RGCs) and star burst amacrine cells (SACs) are

51 known to form regular mosaics and both cell types are widely used to study mosaic organization.

52 SACs are divided into two populations, one located in the inner nuclear layer (INL), and the other

53 in the ganglion cell layer (GCL). Each population forms an independent mosaic.

54 RGCs are located in the GCL and are the output cells of the retina, sending all visual information

55 processed in the retina to the brain visual areas. There are many ways RGCs can be classified into

56 subgroups. One simple, basic approach is to classify them into three functional and morphological

57 groups depending on the sub-layer their dendrites laminate into in the inner plexiform layer (IPL),

58 forming the On, Off and On-Off groups. On cells respond to increase of light while Off cells

59 respond to a decrease of light and On-Off cells respond to both increase and decrease of light.

60 RGCs can however be divided into more than 40 types (1-3), each having different functional and

61 anatomical characteristics. Their density is also known to greatly differ, varying from less than 50

62 cells $/ \mathrm{mm}^{2}$ to more than 300 cells $/ \mathrm{mm}^{2}$ (3). It has been proposed that a group of RGCs has to fulfil

63 four criteria in order to be considered a RGC type (3): 1. Morphological homogeneity (dendritic tree

64 shape). 2. Identical physiological properties (electrophysiological response to light). 3. Similar gene

65 expression (molecular signature). 4. Regular spacing (mosaic). Thus, being organised in mosaics

66 could represent an important feature of each RGC type. Even if the total number of RGC types is

67 estimated to more than 40, only 19 have been fully characterised (cellular density, morphology,

68 molecular signature and functions) (3). Other RGC types have been only partially characterised.

69 RGCs are the first cell class to differentiate in the immature retina, generated in the ventricular

70 zone, followed by migration to the GCL, where they start extending dendrites towards the IPL.

71 RGCs are the only cell class notably more numerous in the immature retina than in the adult retina.

72 Indeed, around 60\% of newly born RGCs undergo programmed cell death (apoptosis) during the 
73 perinatal period (4). Interestingly, not much is known yet about the impact of RGC apoptosis on the

74 maturation of retinal circuitry and visual pathways.

75 Despite being an important feature of retinal organization, retinal mosaic' formation is not fully

76 understood yet. In particular, three mechanisms are believed to potentially take part in their

77 development: cell-fate determination (CF), programmed cell death (CD) and tangential cellular

78 migration $(\mathrm{CM})(2,5)$.

\section{Cell fate determination}

80 CF is a process by which a cell of a certain type will prevent the emergence of same type cells in its

81 immediate vicinity (6). After passing through an intrinsically determined state, retinal progenitors

82 are still left in an undifferentiated state, but are now only capable of giving rise to a limited subset

83 of cell types. The precise type the cells choose to differentiate into depends on extrinsic signals (7).

84 These extrinsic signals can consist of chemical cues such as trans-membrane proteins (8-10) and

85 may be delivered by an already differentiated retinal cell in order to block neighbouring

86 undifferentiated cells from differentiating into the same cell type. This mechanism has been

87 demonstrated in the retina (7) and is believed to be ubiquitous in the developing CNS.

88 Programmed cell death

89 RGCs exhibit a very high rate of programmed death, or apoptosis (60-70\% of the initial population

90 (11)), during normal development. The CD mechanism is believed to be implicated in the selection

91 of relevant cells in order to build a functional retina. Following this principle, cellular death has

92 been proposed to be a consequence of RGCs not being able to establish correct axonal connections

93 in the lateral geniculate nucleus in the thalamus (12). RGC cell death has also been shown to

94 depend on neighbouring cells' electrical activity $(13,14)$. Creating either spatial or functional

95 competition between homotypic cells could lead to the formation or refinement of mosaics. Due to

96 major differences in death rate, the importance of programmed cell death upon mosaic formation

97 seems however to vary between cell classes, and even between subgroups within the same cell 
98 class. Cell death has been proposed to contribute to mosaic formation for several cell groups in the

99 retina, including amacrine cells $(15,16)$ and at least one RGC type (14).

\section{Cellular migration}

101 All retinal cells undergo migration during retinal development, both vertical (from one layer to

102 another) and tangential (horizontal migration within the same layer). Cells can move between 20

103 and $100 \mu \mathrm{m}$ tangentially from their initial location $(17,18)$. This mechanism is believed to be

104 implicated in mosaic formation. This has been demonstrated for SACs mosaic formation, where

105 homotypic cells move tangentially away from each other (19). Tangential migration is believed to

106 be a key mechanism in mosaic formation (15). Mechanisms responsible for this migration are not

107 fully understood, even if chemical cues seem to play a key role, such as in the case of SACs (20).

108 Diffusible signals or contact-mediated interactions between homotypic cells may be responsible for

109 mosaic formation (21). It is important to note that mosaics appear, partially or completely, before

110 extensive dendritic growth $(21,22)$, and thus without contact-mediated interactions. However, other

111 studies point out the importance of dendritic growth upon tangential migration $(20,23,24)$. In all

112 cases, cell-cell interactions seem to be mandatory for tangential migration.

113 Of course, it is likely that the formation of mosaic patterns is due to the combinations of all three

114 mechanisms (22). Previous mathematical simulations of retinal mosaic formation have been

115 conducted $(5,13)$. These studies investigated the involvement of the CF, CD and CM mechanisms,

116 suggesting a central role for the CM mechanism. However, these studies are highly abstract and do

117 not mechanistically model retinal mosaic formation, thus limiting their biological relevance. No

118 mechanistic or biologically plausible model of mosaic formation currently exists.

119 Agent-based (AB) simulation is a type of computational model in which each simulation object is

120 an autonomous agent. Despite the absence of any global supervisor, highly complex structures can

121 emerge from local interactions of agents that self-organise $(25,26)$. It is a particularly relevant

122 approach to model biological phenomena where cells exhibit this characteristic as well. Using the 
$123 \mathrm{AB}$ approach would allow the construction of mechanistic and realistic models of retinal mosaic

124 formation.

125 The impact and implications of all mechanisms involved in mosaic formation (cell-fate, cell death,

126 tangential migration) are not fully understood, and much remains to be done in order to establish the

127 detailed mechanisms governing mosaic formation. In this work, we analyse mechanisms underlying

128 retinal mosaics self-organisation using $\mathrm{AB}$ computational modelling. In particular, the biological

129 requirements and the effect of individual mechanisms generating these cellular patterns are

130 investigated.

\section{Results}

\section{RGC mosaic development}

133 We demonstrate here that a realistic $\mathrm{AB}$ implementation of the CF is able to significantly increase

134 the mosaic regularity compared to a random distribution $(\mathrm{p}<0.001)$. Indeed, as shown in Figure

$1351 \mathrm{~A}$, the average regularity index (RI, used to assess the regularity of the mosaics) values rapidly

136 increase from random levels (between 1.8 and 2) until reaching a value of $2.42( \pm 0.09)$ at the end of

137 the CF mechanism. However, such RI values are lower than the experimentally observed values (>

138 3), and so cannot be considered as solely responsible for the formation of regular mosaics.

139 Moreover, we find that the CF mechanism alone cannot explain high RI scores observed for some

140 RGC types (> 5). As shown in Figure 1B, if CF is the only simulated mechanism, no correlation can

141 be established between cell density and final RI values (correlation magnitude of 0.31 ). Thus,

142 mosaics of high cell density reach similar RI values as seen in mosaics of low cell density, as

143 illustrated by the blue and orange lines in Figure 1A. 

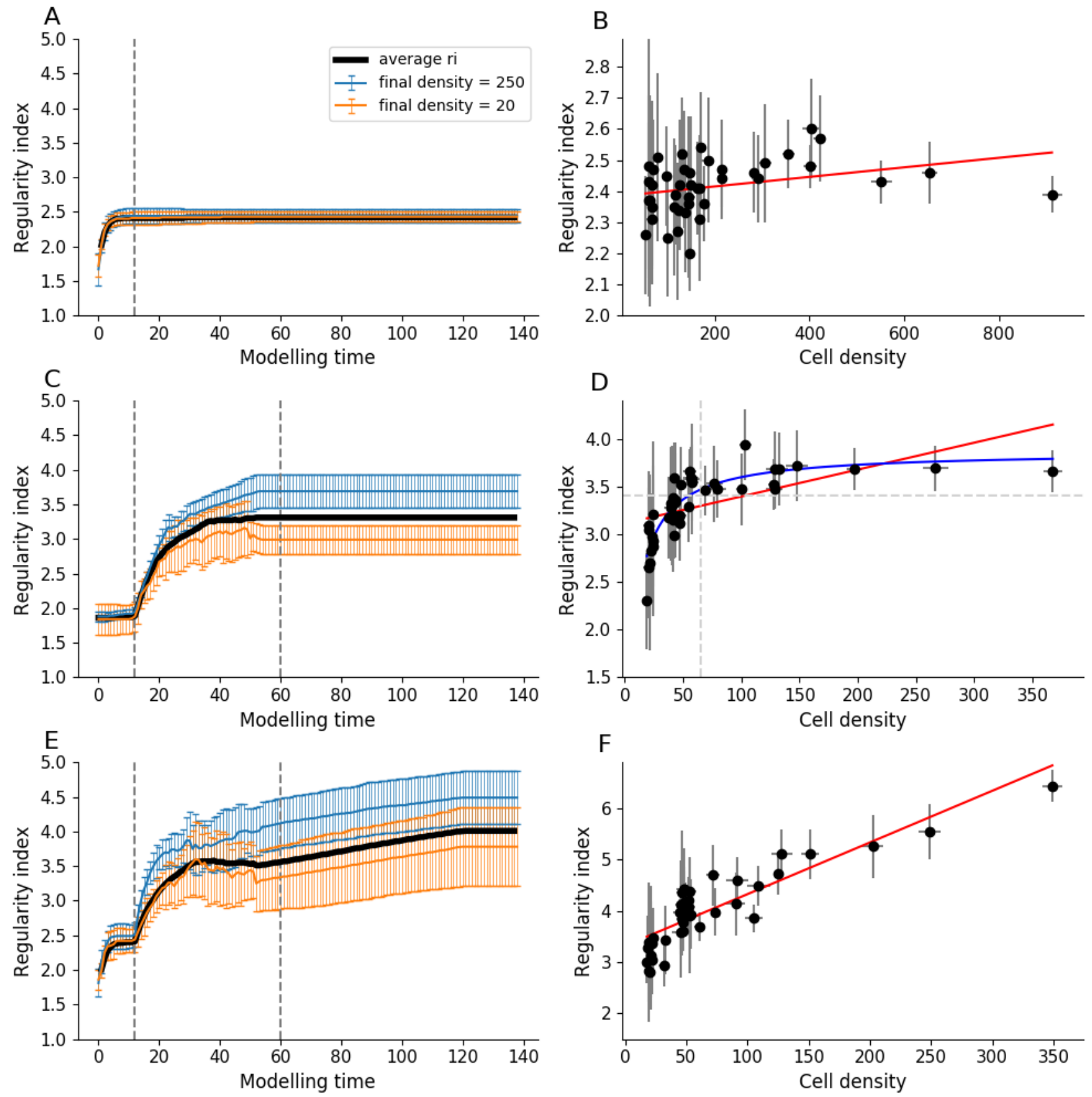

Figure 1: RGC mosaic formation modelling using an ABM approach. A,C,E: RI score evolution during simulation (x axis: 10 visualisation steps correspond to 1 developmental day in mouse). Average RI values for all RGC types are displayed in black while two populations of high and low densities (250 and 20 cells/mm 2 respectively) are displayed in blue and orange. The first vertical grey dashed line on the left indicates, if implemented, the end of the CF mechanism and if implemented, the beginning of the CD mechanism. The second grey dashed line indicates the end of the CD mechanism. B,D,F: Final RI score depending on cell density at the final step of the simulation. Error bars represent standard deviations for average RIs and densities. Red lines represent linear regressions (correlation coefficient: $r=0.31, r=0.58$ and $r=0.87$ for $\mathrm{B}, \mathrm{D}$ and $\mathrm{F}$ respectively). The blue line in $\mathrm{D}$ represents a non- linear regression $\left(\mathrm{a}^{*} \mathrm{x} / \mathrm{b}+\mathrm{x}\right)$, while the horizontal dashed line represents the RI value under which no cell type of density higher than 125 is observed. A,B: CF mechanism only. C,D: CD mechanism only. E,F: Combination of CF, CD and CM combination. 
158 The CD is also able to significantly increase RI compared to a random distribution $(\mathrm{p}<0.001)$, alone

159 or in combination with the CF mechanism. As shown in Figure 1C, the average RI value increases

160 from random (around 1.8$)$ to $3.31( \pm 0.33)$ at the end of CD. This death rate amounts to around $65 \%$

161 when it reaches a steady state at the end of the simulation. These death rate dynamics are very

162 similar to rates observed in-vitro (see Figure 2A). Moreover, and unlike for the case of the CF

163 mechanism, CD is able to generate mosaics of medium regularities ( $\mathrm{RI}>3$ ).

164
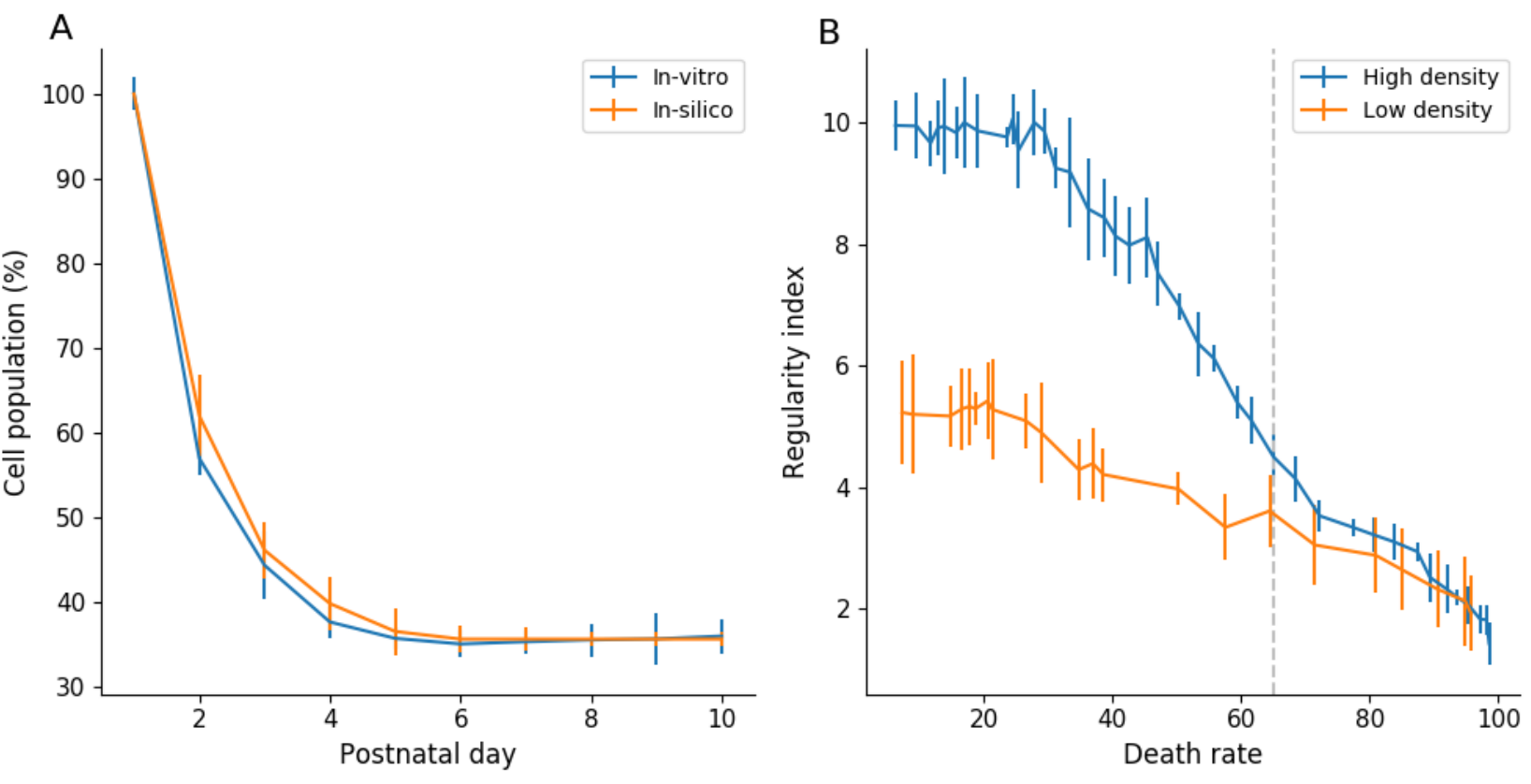

Figure 2: CD mechanism impact on RGC population. A: RGC population measured in-vitro (blue) and in simulations (orange). In-vitro population at day 1 is an estimation based on a final CD of $65 \%$. Error bars represent standard deviation. B: RI score depending on final CD rate in a simulation implementing only the CD mechanism, for selected RGC populations of high density (blue curve, initial density $=571$ cells $/ \mathrm{mm} \mathrm{2}$ ) and low density (orange curve, initial density $=114$ cells $/ \mathrm{mm}^{2}$ ). The vertical dotted line indicate a death rate of $65 \%$. Error bars represent standard deviation.

Interestingly, and as shown by Figure 2B, the death rate measured in-vitro and selected for our simulations (grey vertical dashed line) is not the one generating the highest regularity. Indeed, the highest scores of RI are achieved for death rates between 5 and 30\% of the RGC population, regardless of the initial density of the considered population. After $30 \%$ of cell death, RI decreases until it reaches a random distribution once around $90 \%$ of cell death is achieved. This is observed under both high and low-density conditions. This is observed in simulations with CD alone or in 
178 combination with CF. Interestingly, and in contrast to the CF mechanism, we observe strong

179 differences between populations of high and low initial densities. As shown in Figure 1B, the high-

180 density population is able to generate more regular mosaics than the population of low density, both

181 for their maximum value (RI > 9 and RI > 5, respectively, when cell death is below 30\%) and at

$18265 \%$ of cell death $(\mathrm{RI}=4.49 \pm 0.36$ and $\mathrm{RI}=3.61 \pm 0.59$, respectively). Therefore, a positive

183 correlation between cell density and the final regularity is observed when the death rate is set to

184 65\%. Mosaics of low density exhibit low RI values, while those of cell density higher than 65

185 cells $/ \mathrm{mm}^{2}$ (vertical dashed line of Figure 1D) exhibit a higher average RI score of 3.35 (horizontal

186 dashed line of Figure 1D).

187 While no differences are observed in RI scores between simulation of the CD mechanism and a

188 combination of the CF and CD mechanisms if all mosaics are considered (3.31 \pm 0.33 and 3.48

$189 \pm 0.44$ respectively, $\mathrm{p}=0.76$ ), a positive impact on dense mosaics' regularity (for cell densities

190 higher than 125 cells $/ \mathrm{mm}^{2}$ ) is to be noted. Thereby, RI values in the case of CF and CD combination

191 plateaus around 4.1 instead of 3.6 if CD is the only implemented mechanism.

192 A combination of all three mechanisms (CF, CD and CM) is also able to generate mosaics

193 significantly more regular than random distributions $(\mathrm{p}<0.001)$. Different steps of a simulation are

194 illustrated by Figure 3. 


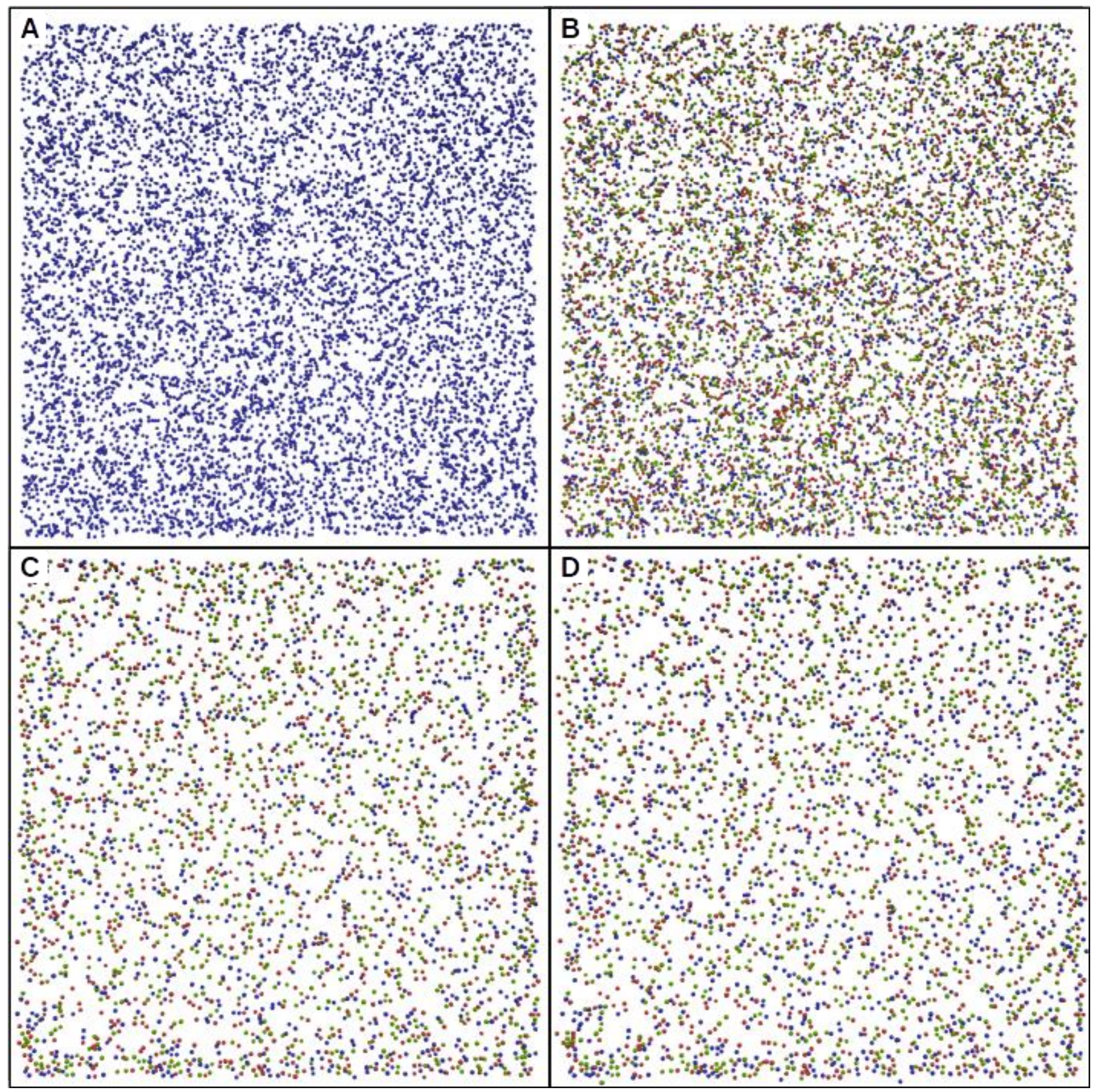

196 Figure 3: Time lapse of mosaic formation, using a combination of CF, CD and CM

197 mechanisms using BioDynaMo. A: Simulation at step 0. All cells are undifferentiated and

198 represented in blue. B,C,D: On cells are represented in green, Off cells in red and On-Off cells in

199 blue. B: Simulation after the end of CF mechanism, at step 180. Average RI = 2.41. C: Simulation

200 at the end of CD mechanism, step 1000. Average RI = 3.42. D: Simulation at the end of CM

201 mechanism, step 2240. Average RI = 3.99.

202 As the CD and CM mechanisms require cells to be differentiated, CF is simulated beforehand. A

203 first RI increase corresponding to the effect of CF is observed (see Figure 1E). After the CD and

204 CM mechanisms are triggered (first dashed line), they give rise to a significant second increase,

205 until the RI value stagnates toward the end of CD (simulation day 4 to 5.5 depending on the cell 
206 type). Finally, a third RI increase is observed after CD is over (second dashed line) due to the CM

207 mechanism, leading to an average RI score of $4.01( \pm 0.75)$ at the end of the simulation. Unlike any

208 other mechanism alone, and thanks to tangential migration, this simulation condition is able to

209 generate highly regular mosaics (RI > 5). Moreover, a strong correlation appears between cell

210 density and RI values (linear correlation magnitude of 0.87 ) as shown by Figure 1F. Thereby, only

211 RGC types exhibiting a cell density higher than 125 cell $/ \mathrm{mm}^{2}$ are able to generate mosaics with a RI

212 value higher than 5. Thus, as illustrated by the blue and orange lines in Figure 1E, significant

213 differences emerge between mosaics of high and low density. No significant differences are seen

214 between simulations of CD and CM combination and simulations of CF, CD and CM combination.

215 When all three mechanisms are implemented, surviving cells migrate tangentially with an average

216 distance of $8.72 \mu \mathrm{m}( \pm 0.11, \mathrm{n}=8$ simulations $)$, which is in accordance with in-vivo measurements

217 reporting migration distance below 30 $\mu$ m (22). Important disparities in migration distance between

218 cells are to be noted, as shown in Figure 4A, with an average migration distance standard deviation

219 of $9.44( \pm 0.18)$. No correlation between final RI and migration distance can be seen. Likewise, no

220 correlation appears between final density and migration distance if the whole population is

221 considered. However, if only populations with a final density higher than 100 cells/mm 2 are

222 considered, a strong correlation can be observed (correlation coefficient $\mathrm{r}=0.92$, see Figure 4B red

223 line). Hence, the denser the cell type the larger the distance cells migrate. 

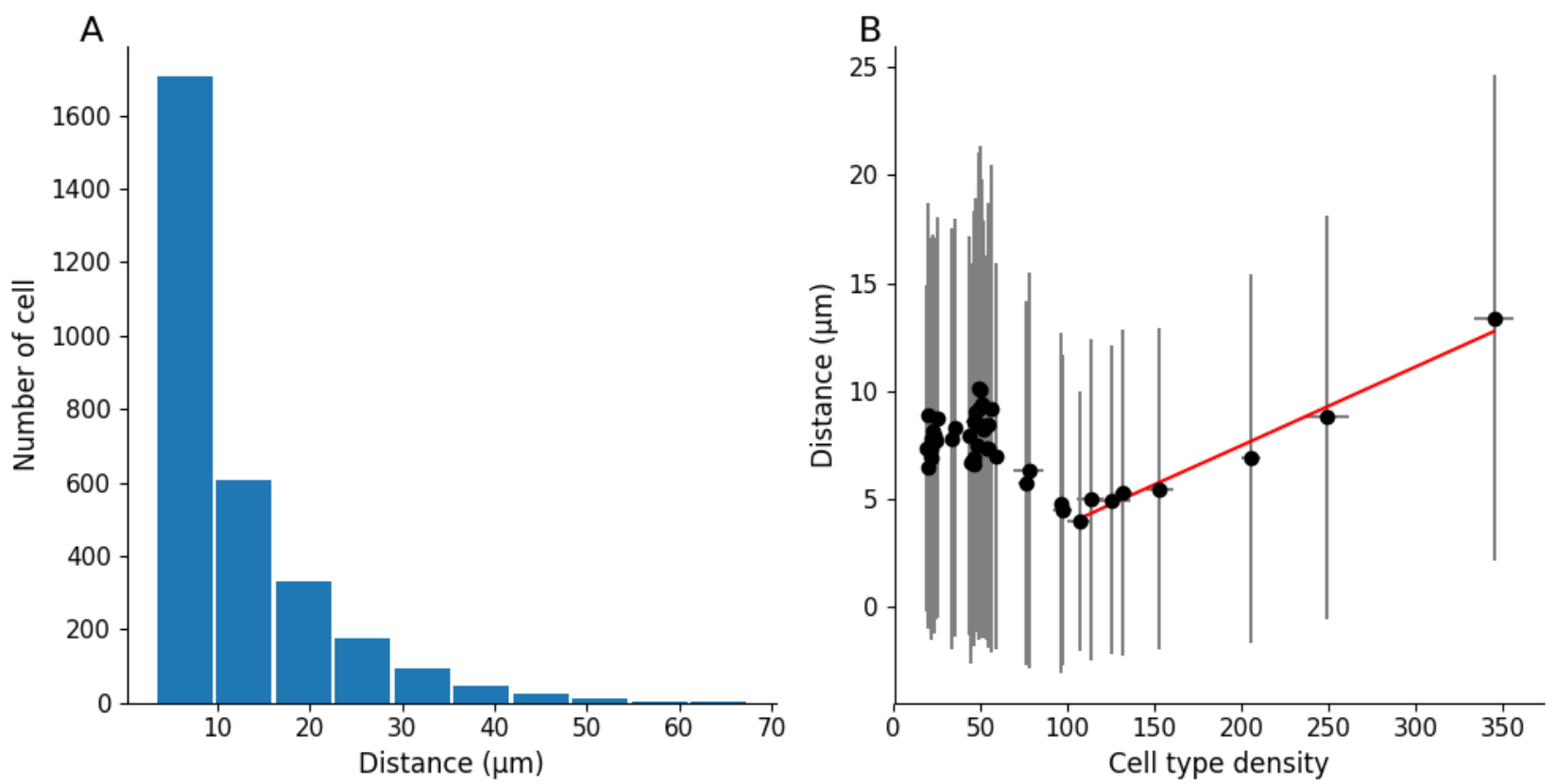

Figure 4: Migration distance measured in simulations implementing CF, CD and CM. A:

Migration distance distribution. B: Relation between cell type density and migrating distance. The red line represents the correlation between migration distance and cell type density for densities higher than 100 cells/mm 2 (correlation coefficient $r=0.92$ ).

\section{$\underline{\text { SAC mosaic development }}$}

The SAC population is divided between two different cellular layers, the GCL and the INL, forming two separate populations (see Figure 5 for an illustration). Our In-vitro results exhibit no significant differences in the GCL and INL populations densities from P4 to P10 ( $\mathrm{p}=0.27$ and $\mathrm{p}=0.32$ respectively, see Figure 6A). These two SAC populations exhibit regular pattern organisation, and no significant difference over time of their RI is measured from P4 to P10, as shown by Figure 6B. GCL and INL SAC mosaics are reported to be independent, in line with experimental data showing that SAC populations in the GCL and the INL only moderately overlap $(20,27,28)$. A measure of these populations' exclusion has then been conducted, showing no significant difference from P4 to P10, as shown by Figure 6C. This indicates that the INL and GCL SAC populations have already

239 formed regular structures from P4 (shortly after GCL and INL separation), and do not exhibit 240 further significant cell migration. This could indicate that mosaics are already formed and do not 

perpetuity. It is made available under aCC-BY 4.0 International license.

241 improve their regularity once SACs have migrated to their respective cellular layer. For this reason,

242 SAC mosaics formation is implemented before GCL/INL separation in our simulations.
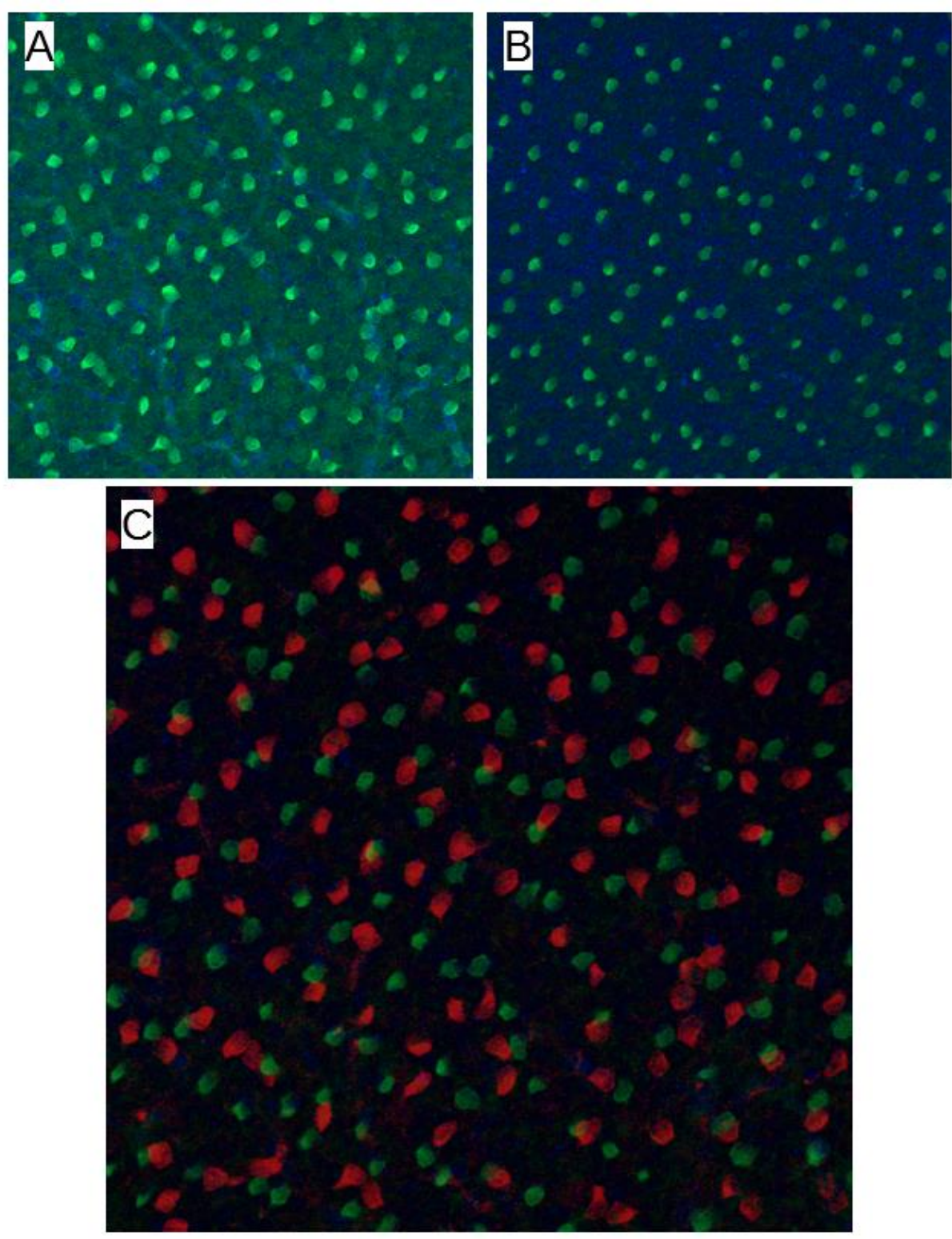

244 Figure 5: ChAT immunostaining on a P9 pup retina. A: GCL level. B: INL level. C: overlap of 245 GCL (red) and INL (green) levels. GCL and INL level images are taken at the same x,y position, but 246 at different depth focus. Regular SACs positioning can be observed in each cellular layer. Few cells 247 overlap between GCL and INL levels are noted. 

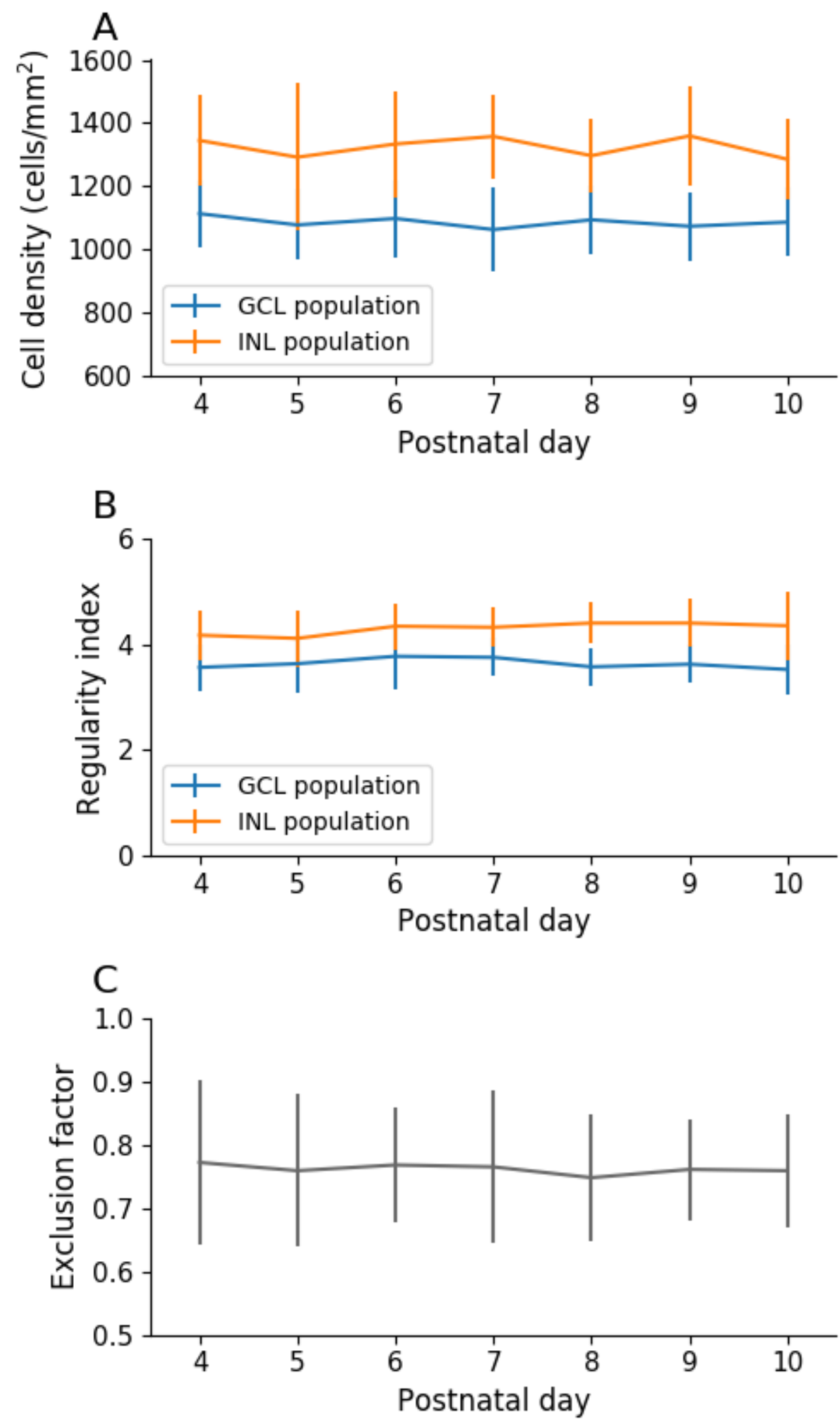

248 Figure 6: In-vitro SAC population characteristics through development. A,B: SACs in the CGL 249 population are represented in blue, and the INL population is represented in orange. A: Cell density 250 over time. B: Regularity index over time. C: GCL and INL SAC population exclusion. A score of 2511 denotes two mosaics with a perfect exclusion and a score of 0 a total overlap of mosaics.

252 Exclusion diameter of $32 \mu \mathrm{m}$. Error bars represent standard deviation. P4: $n=14$; P5: $n=15$; P6:

253 n=14; P7: $n=12$; P8: $n=10 ;$ P9: $n=9$; P10: $n=10$. No differences are to be noted between P4 and P10. 
254 Interestingly, by using an identical concentration threshold triggering CM for SAC in the GCL and

255 INL, the GCL SAC population exhibits less regular mosaics than the INL population at the end of

256 the simulation. This is observed in both developmental conditions, using either one common or two

257 separate (one for the GCL population, one for the INL population) chemical substances for mosaic

258 formation (RI of $3.57 \pm 0.12$ and $4.11 \pm 0.12$ respectively when one substance is used, RI of $3.36 \pm 0.07$

259 and $4.37 \pm 0.15$ respectively when two substances are used, $\mathrm{n}=8$ for each group, $\mathrm{p}<0.0001$ ). This

260 mosaic regularity disparity is in accordance with observations in mouse (see Figure 6B) where the

261 INL population has been reported to be more regular than in the GCL population. In our

262 simulations, this disparity can be explained by the cell density difference between these two layers.

263 Indeed, and as previously demonstrated in our simulations, the denser a cell population is, the more

264 regular its mosaic can be. Hence, our model provides a mechanistic explanation for this observed

265 difference in RIs between the two SAC populations.

266 However, we find that an important difference emerges between the two conditions concerning the

267 exclusion factor of the two SACs populations: if one common developmental cue is used, GCL and

268 INL mosaics exclude each other with a calculated exclusion factor of $0.71( \pm 0.01, n=8)$, similar to

269 what has been measured in-vitro $(0.74 \pm 0.09, \mathrm{n}=5$, see Figure 6$)$. This indicates that the GCL and

270 INL populations’ mosaics tend not to overlap, and so are not fully independent of each other.

271 However, if two distinct developmental cues are used, the exclusion factor is lower, at $0.31( \pm 0.1$,

$272 \mathrm{n}=8$ ), denoting independent mosaics that tend to overlap. In this second condition, the measured

273 exclusion factor is significantly lower than the one observed in mouse $(\mathrm{p}<0.0001$ using a T-test for

274 two independent samples, $\mathrm{n}=8$ and 5 respectively). Thus, only the first condition is able to

275 reproduce the results observed in-vitro.

\section{Discussion}


277 All computational simulations must be built upon biological data in order to offer relevant insight of

278 a scientific problem. For this reason, information about retinal development has been gathered using

279 in-vitro experimental observations. Thus, we followed RGCs characteristics through development

280 using RBPMS staining in neonatal mouse retinas. This allowed us to measure death dynamic from

281 P2 to P11. Using biological data from ours in-vitro experiments and from the literature, we built

282 realistic simulations of retinal cells self-organization. This includes the number of RGCs types

283 incorporated in our simulations, based on evidence from the literature (1-3). Notably, Sanes and

284 Masland (2015) speculated that known RGC types represent only about 60\% of the total RGC

285 population, corresponding to around 1740 cells $/ \mathrm{mm}^{2}$ from the total 3000 cells $/ \mathrm{mm}^{2}$ observed in the mouse retina. In addition, it is important to note that from these known RGC populations, only

$12.4 \%$ are On type. As On, Off and On-Off are equally numerous (30\% to 35\% each), a great number of On cells still needs to be discovered in order to reach the theoretical percentage of On RGC in the total RGC population. Thus, we can hypothesize that either: 1 . Several high density On types have not yet been discovered. 2. There are more On types than Off or On-Off types.

291 The first hypothesis appears unlikely as RGCs are widely studied, especially with the emergence of

292 large-scale and high density MEA recordings, but also using morphological and molecular

293 characterisations. Thus, it is unlikely that the existence of dense On RGC types (representing the

294 majority of the On population, and so being the most common On type) has not been captured by at

295 least one of these techniques. The second hypothesis appears to be supported by experimental

296 evidences because mice, similarly to other nocturnal animals, have rod-dominated vision. Indeed,

297 rods are known to project their dendrites and to establish synaptic connections only to On bipolar

298 cells, that in turn establish synaptic connections to On RGCs. In order to extract as many features as

299 possible from a visual scene using mainly rod vision, a great diversity of specialized RGCs can be

300 justified. The hypothesis of a great diversity of low density On types is also in agreement with

301 Masland et al., (2015), who speculate that around 30 low density RGC types exist and are yet to be

302 discovered. Baden et al. (2016) also estimate the total number of RGC types to be over 40, 
303

304

305

306

307

308

309

310

311

supporting the hypothesis of numerous low density RGC types, including On types. As it is still possible that On types of mid density has not been discovered, we chose to allow the possibility for this hypothesis in our simulations, in addition to adding multiple low density On RGCs.

One major basis of our simulations is the presence of chemical cues supporting cellular selforganization mechanisms. Evidences of such chemical cues have been previously reported $(20,29,30)$.

\section{$\underline{\mathrm{RGC} \text { mosaic formation }}$}

CF implication on RGC mosaics' regularity is particularly difficult to study in-vitro or in-vivo as RGC progenitor cells do not express RGC type-specific markers cells will differentiate into. Despite experimental studies on RGC progenitors, no evidence has been found for RGC type-specific progenitors (31). Hence, RGC types are probably not pre-determined early on and so are likely to depend on extrinsic factors, such as the presence of chemical cues (7). Thereby, it allows for the contribution of a mechanism such as CF for RGC type differentiation, and its potential implication in mosaic formation. One major conclusion from our simulations is that highly regular mosaics (RI > 2.5) cannot be explained only through the CF mechanism. Likewise, the CF mechanism does not significantly increase the power of other mechanisms (CD and/or CM) neither. This suggests that RGC types are unlikely to be defined by cell body mosaics. They may instead be dictated by intrinsic factors (that still remain to be discovered), functional determination (dictated by the input from other cells), or a combination of intrinsic factors interacting with extrinsic factors.

In our simulations, the CD mechanism (alone, or in combination with the CF mechanism) is able to create regular mosaics $(\mathrm{RI}>3.5)$ with a death rate of $65 \%$. As this mechanism is based on a locally diffused chemical substance, homotypic cellular spacing (and therefore cell type initial density) has an important impact on the CD mechanism. For this reason, only populations with a high initial cell density exhibit regular mosaics. Importantly, our CD implementation is able to match measured RGC death dynamics during development, thus strengthening its plausibility. It should be pointed 
out that CD serves additional purposes in the retinal maturation process and is not only geared

towards mosaic creation. Indeed, some cell types which do exhibit mosaic regularity do not undergo

any significant levels of CD (such as horizontal cells or photoreceptors). In addition, as

demonstrated here, the maximum positive impact of $\mathrm{CD}$ upon $\mathrm{RI}$ is reached at death rate lower than

$30 \%$, which is below the $60-70 \%$ death rate observed in mouse. This implies that even if CD can be

involved in mosaic formation at early stages, cell death at levels above $30 \%$ is likely to be driven by

other mechanisms and for other purposes than mosaic regularity. For instance, CD could be

implicated in refining retinal functional connectivity and activity. CD could also have evolutionary

advantage with regard to generating an optimised neural architecture (32).

337 Finally, CM is the only mechanism able to explain the formation of highly regular mosaics $(\mathrm{RI}>5)$.

338 As for the CD mechanism, the efficacy of the CM mechanism is dependent on cell density as it is based on local interactions. The shorter homotypic cellular distances are, the more they can sense and repulse each other. Thereby, a strong correlation emerges between RGC type populations densities and the regularity of their mosaics. Therefore, we propose here that low density RGC type mosaics exhibit on average significantly lower regularities than high density RGC types mosaics. It would be very informative to experimentally verify this prediction. To this date, this question

344 remains unanswered. This hypothesis is in accordance with recent studies showing that some low

345 density RGCs do not exhibit regular spacing (33). Moreover, we question here the relevance of 346 regular spacing as a criterion for a group of RGC to form a RGC type. Indeed, if all low density

347 RGC types do not exhibit highly regular spacing as predicted here, this criterion does not

348 discriminate RGC types.

349 We show here that high mosaic regularity can be achieved with limited migration distance $(8.72 \mu \mathrm{m}$

$350 \pm 0.11$ in average, $n=8$ ). This average migration distance is in accordance with in-vivo

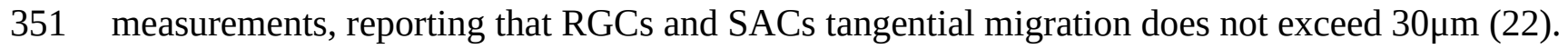

352 However, the average migration distance measured in our simulations is notably lower than the 
353 average migration distance experimentally measured at around $20 \mu \mathrm{m}$ (19) and could be explained

354 by the absence of retinal surface expansion implementation in our simulations. The CM mechanism

355 implemented here is based only on local cues and short-distance interactions, and thereby follows

356 the description of tangential dispersion in mouse, reported as a local, short-distance, phenomenon

357 (21). Our results are consistent with previous studies showing that a tangential cell dispersion does

358 not appear to be directly related to the cell time of birth, but rather to its cell type (21).

359 The cellular migration and RI dynamics resulting from the CM mechanism are in agreement with

360 the literature, where it is reported that RI increases mostly between P1 and P5, with the spacing

361 between cells still increasing after that period, until P10 (2). After reaching the correct cell layers, a

362 slower and finer tangential positioning phase of RGC within the GCL has been reported $(19,34)$.

363 Cellular movement during this period has been described as random but important for exact cellular

364 positioning (34). In accordance with our results and as stated by other studies (27), these highly

365 varied movements are likely to be related to mosaic formation and refinement. Indeed, these

366 movements appear random as the whole RGC population (On, Off, On-Off population) is

367 considered, while RGC populations should themselves be divided into types in order to

368 meaningfully investigate RGCs lateral migration. If it were possible to examine each type

369 independently, our model suggests that these movements, reported as random, would appear as

370 coherent, as illustrated by Figure 7. 
A

372 Figure 7: Cellular migration appearing as random if the whole population is considered homogeneously (A) or coherent (homotypic avoidance) if the population is sub-divided into two population (B).

\section{$\underline{\text { SAC mosaic formation }}$}

Beside most of RGCs, other retinal cell types are known to exhibit regular spacing, including SACs. This cell population is divided into the GCL and the INL. Both SAC layers form mosaics, that are reported to be independent from each other. Thus, there are only few overlaps between their populations. We found no RI variation from P3-4, indicating that these two SACs populations have already created their mosaics by P3-4, hence shortly after SACs migration into their respective cellular layer. In addition, we observed that the calculated exclusion factor does not vary, also supporting this assumption. Moreover, the observed complementarity of GCL and INL mosaics perhaps indicates interactions between these two SAC populations during their cellular organisation, before they migrate to their respective layer.

Here, we investigated this GCL/INL population interaction hypothesis further by building a simulation of SACs mosaics development. These simulations clearly show that our modelling procedure can successfully be applied to another cell population, without changing any simulation parameters. Indeed, we have been able to explain differences in GCL and INL mosaic regularities
B

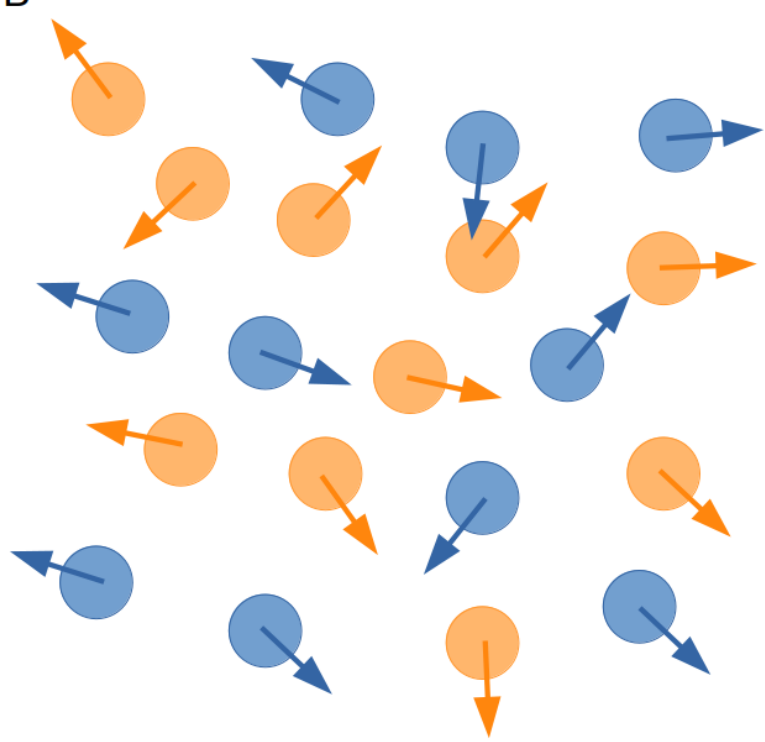


389 (the RI of the INL population being higher than that of the GCL population) by using only local

390 interactions between SACs. This is the case if SACs constitute a unique population, or if GCL and

391 INL populations are distinct (in other words, if one common or two distinct chemical cues are

392 used). In the former case, this RI difference can be explained by the higher number of cells

393 migrating to the INL compared to the GCL. Precisely, the percentage of a population characterised

394 by a highly regular mosaic dictates the regularity of the resulting sub-population. Hence, the bigger

395 the sub-population, the closer the obtained RI will be to the RI of the initial population, if cells

396 constituting this sub-population are chosen randomly. For instance, if a population with a high RI is

397 randomly divided into two sub-populations of $80 \%$ and $20 \%$ of the initial population (denoted

398 respectively sub-population A and B), the sub-population A will have a RI closer to the initial

399 population than the sub-population B. Thus, in the latter case, this observed RI difference between

400 the GCL and INL populations can be explained by the higher cell density of SACs in the INL. This

401 higher cell density in the INL allows more interactions and homotypic repulsion and thus the

402 emergence of a higher RI than for the cells located in the GCL.

403 However, and importantly, only the simulation condition using a common chemical cue for mosaic

404 formation is able to explain the complementarity observed between the GCL and INL populations.

405 Indeed, if the two mosaics (GCL and INL) are formed independently, they largely overlap without

406 exhibiting the mutual exclusion observed in-vitro. This suggests that the GCL and INL populations

407 of SACs are not fully independent. Hence, our results predict that a shared guidance cue is

408 responsible for mosaic formation of SACs in the GCL and INL. Locally diffused chemical

409 (molecular) guidance could be a possible cue candidate for mosaic formation. If this is the case, our

410 prediction could be potentially experimentally verified by using knock-out experiments blocking

411 either the secretion or the reception of this chemical guidance.

\section{Methods}

\section{Experimental work}




\section{Immunohistochemistry}

415 Retinal wholemounts were prepared from mouse pups aged P2-P11, flattened on nitrocellulose

416 membrane filters and fixed for $45 \mathrm{~min}$ in 4\% paraformaldehyde. Retinas were then incubated in

417 blocking solution - consisting in $5 \%$ of secondary antibody host species serum with $0.5 \%$ Triton

$418 \mathrm{X}-100$ in $0.1 \mathrm{M}$ phosphate buffer solution (PBS) - for 1 hour.

419 Retinas were incubated with 0.5\% Triton X-100 with RBPMS (1:500) and ChAT (1:500) in PBS for

4203 days at $4^{\circ} \mathrm{C}$, then washed with PBS and incubated with $0.5 \%$ Triton X-100 with donkey anti rabbit

421 Alexa 568 (1:500) and donkey anti goat Dylight 488 (1:500) in PBS for 1 day at $4^{\circ} \mathrm{C}$. Finally,

422 retinas were washed with PBS and embedded with OptiClear. Primary antibodies used were ChAT

423 (AB144P, goat polyclonal, Merck Millipore) for SACs staining and RBPMS (1830-RBPMS, rabbit

424 polyclonal, Phosphosolutions) for RGCs staining. Secondary antibodies used were Donkey anti

425 rabbit Alexa 568 (A10042, Invitrogen) and Donkey anti goat Dylight 488 (SA5-10086,

426 ThermoFisher Scientific).

427 Zeiss AxioImager with Apotome processing and the Zeiss LSM 800 confocal microscope were used 428 to image the retinas. High-resolution of the whole retinal surface was achieved by imaging multiple

429 individual adjacent areas. Individual images were subsequently stitched back together to view the

430 entire retinal surface. Images at 40x magnification were also acquired in mid-peripheral regions in

431 order to perform cell count and mosaic regularity measures.

432 Cell populations density

433 The average RGC and SAC density for each developmental day was measured by performing a

434 manual cell count from P2 to P10 for RGCs and from P4 to P10 for SACs. By accounting for the

435 surface expansion observed during retinal development, we estimated changes in populations

436 through development. The estimated total RGC and SAC populations for a given retina are

437 calculated by multiplying the averaged cell density (obtained from 3-6 sample areas per retina) by

438 its corresponding retinal surface. These individual measurements are then averaged for each 
439 developmental day to give an estimation of the total population from P2 to P10. Cell population

440 death rate during development is then calculated. In detail, the population of each retina measured

441 on day $\mathrm{D}+1$ is subtracted from the population of each retina measured on day D to calculate the

442 amount of CD between day D and D+1. The amount of apoptosis measured between two

443 consecutive days is then averaged to calculate the daily death rate of RGC and SAC populations.

444 SAC populations in the GCL and INL are calculated separately.

$445 \underline{\text { SAC mosaics }}$

446 Positions of SACs in the GCL and INL are also extracted in order to calculate mosaic regularities of

447 these two populations from P4 to P10. A measure of GCL and INL mosaics exclusion has also been

448 conducted. The calculated exclusion factor is based, for two distinct populations, on a count of cells

449 from the first populations located within a determined distance (exclusion diameter) from cells

450 belonging of the second population. This score is then normalised, to give an exclusion factor

451 between 0 and 1.1 denotes a perfect exclusion, meaning that all cells of the first population are

452 located at a distance greater than the exclusion diameter from all cells of the second population. By

453 consequence, only exclusion factors calculated with an identical exclusion diameter can be

454 compared. A unique exclusion diameter of $32 \mu \mathrm{m}$ has been chosen here, corresponding to about 3

455 times the diameter of a SAC soma, and allowing a good discrimination between our different

456 mosaics.

457 Ethics Statement

458 The experimental work was approved by the Animal Welfare Ethical Review Board (AWERB) of

459 Newcastle University.

460 BioDynaMo

461 Simulations were conducted using the agent-based simulation framework BioDynaMo (35). 
462 Each object in BioDynaMo is denoted as a simulation object, and possesses its own characteristics,

463 such as its 3D geometry, mass, adherence and position in space. Individual neurons are represented

464 by a sphere. Diffusion in 3D of chemical substances in the extracellular space has also been

465 implemented, with the discrete central difference method. This diffusion is supported by grids

466 representing substances concentration and gradients. Mechanical forces are also taken into account

467 between all simulation objects such that they cannot overlap, but mechanically repulse each other.

468 Each simulation object can have a biology module attached to it, that describes its behaviour at each

469 simulation time step, such as substance secretion, cell migration or cell growth.

470 As an $\mathrm{AB}$ simulation framework, each simulation object is independent, without a central

471 organisation unit that orchestrates the behaviour of all simulation objects. Thus, simulation objects

472 only have access to their micro-environment, which consists of other simulation and chemical

473 substances of the extracellular matrix in their proximity.

474 Several biology modules have been defined and used in our simulations, in order to describe cells

475 behaviour for self-organisation (cell fate, cell death and cell migration) and chemical substances

476 secretion.

\section{Simulations}

478 All simulations took place in a cubic space of $1,300 \mu \mathrm{m}^{3}$, with cells of 7 to $8 \mu \mathrm{m}$ diameter randomly

479 distributed (uniform distribution) in a space of $1,000 \mu \mathrm{m} \times 1,000 \mu \mathrm{m} \times 22 \mu \mathrm{m}$. The initial cell density

480 has been set to 8600 cells $/ \mathrm{mm}^{2}$, in order to reach the RGC density once programmed CD

481 mechanism is over - around 3000cells $/ \mathrm{mm}^{2}$ reported in literature (3) and around 3500cells $/ \mathrm{mm}^{2}$ in

482 our measures. Mechanical interactions between simulation objects are taken into account, such that

483 they cannot overlap, and mechanically repulse each other. The time step is set such that 160 steps

484 simulate one day of development. Mosaic formation simulations run for a maximum of 2240 steps,

485 corresponding to 14 days of development. 
486 The global RGC population is subdivided into 43 types. Some have been precisely documented,

487 such as the On or On-Off direction selective ganglion cells (DSGC), the local edge detector (LED),

488 or the Off J-RGCs, and their population densities and dendritic arbours characteristics are known.

489 However, these precisely documented RGC groups represent only 19 types, and merely about 60\%

490 of the total RGC population ( 1700 cells $/ \mathrm{mm}^{2}$ over $\sim 3000$ cells $/ \mathrm{mm}^{2}$ ) (3). RGC types composing the

491 remaining $40 \%$ of the population have been estimated using results from Sanes and Masland

492 (2015), Reese and Keeley (2015) and Baden et al. (2016). These authors state that numerous RGC

493 types are still unknown and these cells are probably sparsely distributed across the retina. Thus, we

494 implemented 24 additional RGC types of various but low densities. All implemented RGC types

495 and their corresponding starting and final densities are summarised in Table 1. 


\begin{tabular}{|c|c|c|c|c|c|c|c|}
\hline Cell type & Type name & $\begin{array}{l}\text { Start density } \\
\text { cells } / \mathrm{mm}^{2}\end{array}$ & $\begin{array}{c}\text { Final density } \\
\text { cells } / \mathrm{mm}^{2}\end{array}$ & $\begin{array}{c}\text { D } \\
\text { Death }\end{array}$ & $\begin{array}{c}\text { FD } \\
\text { Death }\end{array}$ & $\begin{array}{l}\text { FDM } \\
\text { Death }\end{array}$ & $\begin{array}{c}\text { FDM } \\
\text { Migration }\end{array}$ \\
\hline 0 & on-off_dsgca & 357 & 125 & 2.0367 & 2.0334 & 2.023 & 2.02 \\
\hline 1 & on-off_dsgcb & 357 & 125 & 2.0367 & 2.0334 & 2.023 & 2.02 \\
\hline 2 & on-off_dsgcc & 357 & 125 & 2.0367 & 2.0334 & 2.023 & 2.02 \\
\hline 3 & on-off_dsgcd & 357 & 125 & 2.0367 & 2.0334 & 2.023 & 2.02 \\
\hline 4 & on-off_m3 & 57 & 20 & 1.9872 & 1.9855 & 1.9855 & 1.983 \\
\hline 5 & on-off_led & 714 & 250 & 2.116 & 2.098 & 2.08 & 2.065 \\
\hline 6 & on-off_u & 57 & 20 & 1.9872 & 1.9855 & 1.985 & 1.983 \\
\hline 7 & on-off_v & 57 & 20 & 1.9872 & 1.9855 & 1.985 & 1.983 \\
\hline 8 & on-off_w & 171 & 60 & 2.001 & 1.9978 & 1.996 & 1.994 \\
\hline 9 & on-off_x & 143 & 50 & 1.9968 & 1.9945 & 1.994 & 1.9925 \\
\hline 10 & on-off_y & 114 & 40 & 1.994 & 1.993 & 1.993 & 1.991 \\
\hline 11 & on-off_z & 114 & 40 & 1.994 & 1.993 & 1.993 & 1.991 \\
\hline 100 & on_dsgca & 114 & 40 & 1.994 & 1.993 & 1.993 & 1.991 \\
\hline 101 & on_dsgcb & 114 & 40 & 1.994 & 1.993 & 1.993 & 1.991 \\
\hline 102 & on_dsgcc & 114 & 40 & 1.994 & 1.993 & 1.993 & 1.991 \\
\hline 103 & on_aplha & 114 & 40 & 1.994 & 1.993 & 1.993 & 1.991 \\
\hline 104 & on_m2 & 160 & 56 & 2 & 1.9953 & 1.994 & 1.992 \\
\hline 105 & on_m4 & 57 & 20 & 1.9872 & 1.9855 & 1.985 & 1.983 \\
\hline 106 & on_m5 & 57 & 20 & 1.9872 & 1.9855 & 1.985 & 1.983 \\
\hline 107 & on_o & 428 & 150 & 2.05 & 2.0425 & 2.035 & 2.031 \\
\hline 108 & on_p & 286 & 100 & 2.022 & 2.018 & 2.012 & 2.01 \\
\hline 109 & on_q & 286 & 100 & 2.022 & 2.018 & 2.012 & 2.01 \\
\hline 110 & on_r & 228 & 80 & 2.011 & 2.0082 & 2.004 & 2.002 \\
\hline 111 & on_s & 171 & 60 & 2.001 & 1.9978 & 1.995 & 1.993 \\
\hline 112 & on $\mathrm{t}$ & 171 & 60 & 2.001 & 1.9978 & 1.995 & 1.993 \\
\hline 113 & on_u & 143 & 50 & 1.9968 & 1.9945 & 1.994 & 1.9925 \\
\hline 114 & on_v & 143 & 50 & 1.9968 & 1.9945 & 1.994 & 1.9925 \\
\hline 115 & on_w & 97 & 34 & 1.993 & 1.9934 & 1.989 & 1.987 \\
\hline 116 & on_ $x$ & 57 & 20 & 1.9872 & 1.9855 & 1.985 & 1.983 \\
\hline 117 & on_y & 57 & 20 & 1.9872 & 1.9855 & 1.985 & 1.983 \\
\hline 118 & on_z & 57 & 20 & 1.9872 & 1.9855 & 1.985 & 1.983 \\
\hline 200 & off_aplhaa & 114 & 40 & 1.994 & 1.993 & 1.993 & 1.991 \\
\hline 201 & off_aplhab & 114 & 40 & 1.994 & 1.993 & 1.993 & 1.991 \\
\hline 202 & off_m1 & 180 & 63 & 2.006 & 1.9979 & 1.998 & 1.996 \\
\hline 203 & off j & 571 & 200 & 2.078 & 2.065 & 2.058 & 2.049 \\
\hline 204 & off_mini_j & 1000 & 350 & 2.179 & 2.155 & 2.134 & 2.098 \\
\hline 205 & off_midi_j & 228 & 80 & 2.011 & 2.0082 & 2.004 & 2.002 \\
\hline 206 & off_u & 57 & 20 & 1.9872 & 1.9855 & 1.985 & 1.983 \\
\hline 207 & off_v & 57 & 20 & 1.9872 & 1.9855 & 1.985 & 1.983 \\
\hline 208 & off_w & 171 & 60 & 2.001 & 1.9978 & 1.995 & 1.993 \\
\hline 209 & off_x & 143 & 50 & 1.9968 & 1.9945 & 1.994 & 1.9925 \\
\hline 210 & off_y & 114 & 40 & 1.994 & 1.993 & 1.993 & 1.991 \\
\hline 211 & off_z & 106 & 37 & 1.9935 & 1.9928 & 1.989 & 1.988 \\
\hline
\end{tabular}

497 Table 1: Implemented RGC types and parameters used for different conditions. D: death

498 mechanism only. FD: fate and death mechanisms. FDM: fate, death and migration mechanisms.

499 Death: concentration threshold for death mechanism. Migration: concentration threshold for

500 migration mechanism. Parameters have been empirically chosen.

501 Cells are created with no predefined types when simulating the CF mechanism. Otherwise, cells of

502 each RGC type are created matching their experimentally observed initial density.

503 Substance secretion 
504 Each RGC type secretes a specific chemical substance that diffuses in the extracellular space, using

505 grids of $2 \mu \mathrm{m}^{3}$ voxels. This secretion corresponds to an increase of substance concentration by 1 at

506 the cell centre position. Undifferentiated cells do not secrete any substance.

$\underline{\text { RGC mosaic formation: CF }}$

508 CF is implemented such that substances act as an inhibitor for cell differentiation, preventing

509 nearby undifferentiated cells to adopt the same types. In this way, neighbouring cells are forced to

510 differentiate into other RGC types. CF is the first event to occur during simulations, because CD

511 and CM mechanisms operate on differentiated cells.

\section{RGC mosaic formation: CD}

513 The CD mechanism corresponds to the cells removing themselves from the simulation if their

514 corresponding substance concentration is higher than a defined threshold. In this way, the clusters of

515 homotypic cells exhibit high death rates and become sparser. As the cell density decreases, the

516 initial multilayer collapses into a RGC monolayer. This is implemented as cells moving along the $\mathrm{z}$

517 axis toward the centre of the RGC layer, using their chemical cue. CD is triggered after completion

518 of CF, and continues until a steady-state is reached, around a death rate of $65 \%$. This steady-state is

519 reached without global controllers but depends on the chosen concentration threshold triggering cell

520 death. If this threshold is low the steady-state will be reached with a high death rate, while if this

521 threshold is high the steady-state will be reached with a low death rate.

\section{RGC mosaic formation: CM}

$523 \mathrm{CM}$ is implemented such that the homotypic substances act as a repulsive factor. Thereby, cells

524 exhibit short distance avoidance, moving tangentially against their substance gradient, distancing

525 themselves from homotypic neighbours. We assume that CM is triggered after completion of CF, at

526 the same time as CD, and continues either until a steady state or day 13 is reached. 
527 Development conditions incorporating all combinations of these three mechanisms have been

528 investigated. As the mechanisms influence each other, parameters vary depending on the

529 implemented mechanisms. The CD mechanism parameters were chosen for each RGC type such

530 that its final death rate is about 65\%. The CM parameters were chosen depending on the CD

531 parameter value and such that the interaction is kept to close range distance. Pseudocode

532 corresponding to these three biology modules can be found in Pseudocode 1. Table 1 summarises

533 the parameters used for RGC mosaics formation mechanisms.

A

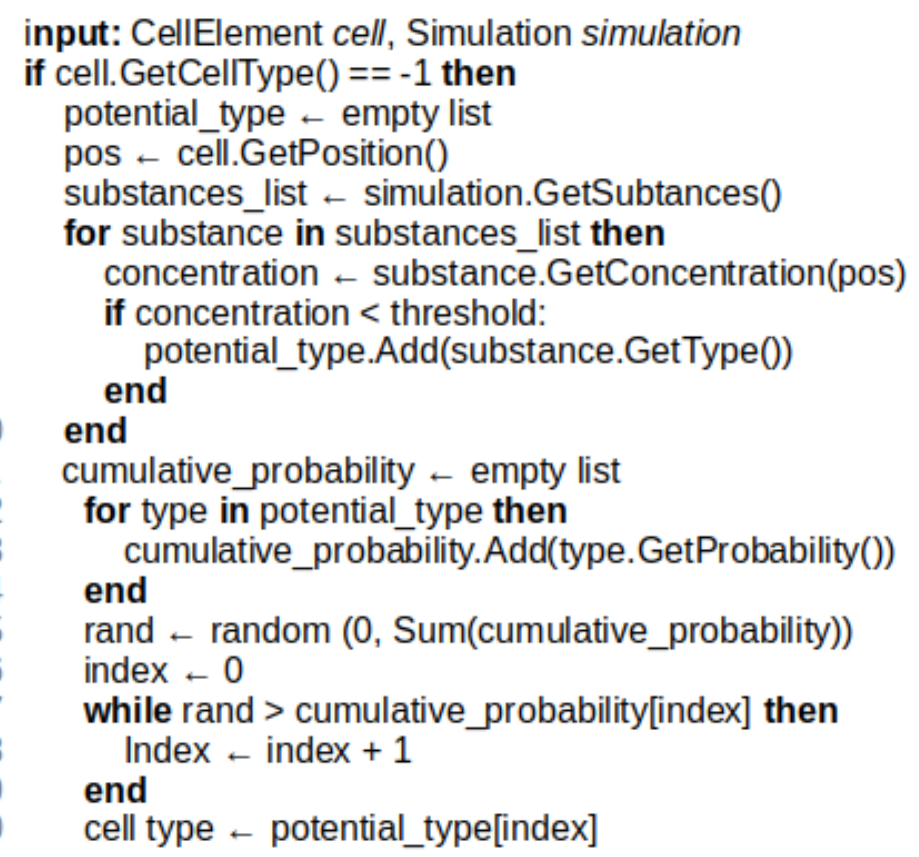

B

input: CellElement cell, ChemicalSubstance substance

pos $\leftarrow$ cell.GetPosition()

concentration $\leftarrow$ substance.GetConcentration(pos)

if concentration > threshold and $\operatorname{Ramdom}(0,1)<$ death_probability then cell.Remove() end

C

input: CellElement cell, ChemicalSubstance substance

pos $\leftarrow$ cell.GetPosition()

concentration $\leftarrow$ substance.GetConcentration(pos)

if concentration > threshold then

direction $\leftarrow$ substance.GetGradient() * reverse_gradient

cell.UpdatePosition(direction)

end

535 Pseudocode 1: Pseudocode describing biology modules. A: cell fate. B: cell death. C: cell 536 migration. 
$\underline{\text { SAC mosaic formation }}$

538 The simulation of SAC mosaic formation is achieved using locally diffused chemical cues

539 triggering homotypic avoidance (tangential migration mechanism). Once mosaics are formed, the

540 two populations migrate to their respective layers, along the $\mathrm{Z}$ axis. Two different developmental

541 conditions have been implemented, using either one common or two separated (one for GCL

542 population, one for INL population) chemical substances for mosaics formation. Importantly,

543 concentration thresholds are identical for the GCL and INL populations. Parameters have been set

544 such that the mosaic RIs match the measured RIs in mouse SACs mosaics.

\section{Data analysis}

546 The RI was used to assess the regularity of the mosaics. It is computed as the average value of the

547 closest neighbour distribution (distribution of the closest neighbour measured for each cell) divided

548 by its standard deviation (36). The RI offers a single score that is able to discriminate regularity

549 differences between mosaics of low regularities. In addition, and as previously reported by Reese

550 and Keeley (2015), the RI offers a scale-invariant measure of mosaic regularity and thus more direct

551 evidence of any change in the mosaic spatial organisation during development. It is not only the

552 absolute RI value that carries information, but also its evolution across development, related to the

553 contribution of each mosaic developmental mechanism (CF, CD, CM). However, it should be

554 pointed out that RI is sensitive to a low sampling rate, leading to significant variability in RI scores

555 for mosaics constituted of few cells.

556 Comparisons between two RI values have been conducted using T-tests for two independent 557 samples.

\section{Author Contributions}

559 JdM, ES and RB conceived and designed the experiments and wrote the paper. JdM performed the 560 experiments and analyzed the data. 
561

562

563

564

565

566

\section{References}

1. Baden T, Berens P, Franke K, Román Rosón M, Bethge M, Euler T. The functional diversity of retinal ganglion cells in the mouse. Nature. 2016 Jan 21;529(7586):345-50.

2. Reese BE, Keeley PW. Design principles and developmental mechanisms underlying retinal mosaics. Biol Rev Camb Philos Soc. 2015 Aug;90(3):854-76.

3. Sanes JR, Masland RH. The types of retinal ganglion cells: current status and implications for neuronal classification. Annu Rev Neurosci. 2015 Jul 8;38:221-46.

4. Farah MH. Neurogenesis and cell death in the ganglion cell layer of vertebrate retina. Brain Res Rev. 2006 Sep;52(2):264-74.

5. Eglen SJ. Development of regular cellular spacing in the retina: theoretical models. Math Med Biol. 2006 Jun;23(2):79-99.

6. McCabe KL, Gunther EC, Reh TA. The development of the pattern of retinal ganglion cells in the chick retina: mechanisms that control differentiation. Development. 1999 Dec;126(24):5713-24.

7. Livesey FJ, Cepko CL. Vertebrate neural cell-fate determination: Lessons from the retina. Nat Rev Neurosci. 2001 Feb;2(2):109-18.

8. Kohwi M, Doe CQ. Temporal fate specification and neural progenitor competence during development. Nat Rev Neurosci. 2013 Dec;14(12):823-38.

9. Rodriguez M, Choi J, Park S, Sockanathan S. Gde2 regulates cortical neuronal identity by controlling the timing of cortical progenitor differentiation. Development. 2012 Oct $15 ; 139(20): 3870-9$.

10. Zhang XM, Yang XJ. Regulation of retinal ganglion cell production by Sonic hedgehog. Development. 2001 Mar 15;128(6):943-57.

11. Finlay B, Pallas S. Control of cell number in the developing mammalian visual system. Progress in Neurobiology. 1989;32(3):207-34. 
586 12. Jeffery G. Retinal ganglion cell death and terminal field retraction in the developing rodent visual system. Brain Res. 1984 Mar;315(1):81-96.

588 13. Eglen SJ, Willshaw DJ. Influence of cell fate mechanisms upon retinal mosaic formation: a modelling study. Development. 2002 Dec;129(23):5399-408.

14. Jeyarasasingam G, Snider CJ, Ratto GM, Chalupa LM. Activity-regulated cell death contributes to the formation of ON and OFF alpha ganglion cell mosaics. J Comp Neurol.

15. Hoon M, Okawa H, Della Santina L, Wong ROL. Functional architecture of the retina: development and disease. Prog Retin Eye Res. 2014 Sep;42:44-84.

16. Resta V, Novelli E, Di Virgilio F, Galli-Resta L. Neuronal death induced by endogenous extracellular ATP in retinal cholinergic neuron density control. Development. 2005

17. Reese BE, Necessary BD, Tam PP, Faulkner-Jones B, Tan SS. Clonal expansion and cell dispersion in the developing mouse retina. Eur J Neurosci. 1999 Aug;11(8):2965-78.

18. Reese BE, Harvey AR, Tan SS. Radial and tangential dispersion patterns in the mouse retina are cell-class specific. Proceedings of the National Academy of Sciences. 1995 Mar

19. Galli-Resta L, Resta G, Tan SS, Reese BE. Mosaics of islet-1-expressing amacrine cells assembled by short-range cellular interactions. J Neurosci. 1997 Oct 15;17(20):7831-8.

20. Kay JN, Chu MW, Sanes JR. MEGF10 and MEGF11 mediate homotypic interactions required for mosaic spacing of retinal neurons. Nature. 2012 Mar;483(7390):465-9.

607 21. Reese BE, Galli-Resta L. The role of tangential dispersion in retinal mosaic formation. Progress in Retinal and Eye Research. 2002 Mar;21(2):153-68.

609 22. Nguyen-Ba-Charvet KT, Chédotal A. Development of retinal layers. C R Biol. 2014 Mar;337(3):153-9. 
611 23. Galli-Resta L. Putting neurons in the right places: local interactions in the genesis of retinal architecture. Trends Neurosci. 2002 Dec;25(12):638-43.

613 24. Huckfeldt RM, Schubert T, Morgan JL, Godinho L, Di Cristo G, Huang ZJ, et al. Transient neurites of retinal horizontal cells exhibit columnar tiling via homotypic interactions. Nat Neurosci. 2009 Jan;12(1):35-43.

25. Bauer R, Zubler F, Pfister S, Hauri A, Pfeiffer M, Muir DR, et al. Developmental selfconstruction and -configuration of functional neocortical neuronal networks. PLoS Comput Biol. 2014 Dec;10(12):e1003994.

26. Kassraian-Fard P, Pfeiffer M, Bauer R. A generative growth model for thalamocortical axonal branching in primary visual cortex. PLoS Comput Biol. 2020 Feb;16(2):e1007315.

27. Chow RW, Almeida AD, Randlett O, Norden C, Harris WA. Inhibitory neuron migration and IPL formation in the developing zebrafish retina. Development. 2015 Aug 1;142(15):266577.

28. Rockhill RL, Euler T, Masland RH. Spatial order within but not between types of retinal neurons. Proceedings of the National Academy of Sciences. 2000 Feb 29;97(5):2303-7.

29. Edwards MM, Mammadova-Bach E, Alpy F, Klein A, Hicks WL, Roux M, et al. Mutations in Lama1 Disrupt Retinal Vascular Development and Inner Limiting Membrane Formation. Journal of Biological Chemistry. 2010 Mar;285(10):7697-711.

30. Tissir F, Goffinet AM. Reelin and brain development. Nat Rev Neurosci. 2003 Jun;4(6):496-505.

31. Sweeney NT, James KN, Nistorica A, Lorig-Roach RM, Feldheim DA. Expression of transcription factors divides retinal ganglion cells into distinct classes. J Comp Neurol. 2019

634 32. Bauer R, Clowry GJ, Kaiser M. Creative Destruction: A Basic Computational Model of 
636 33. Keeley PW, Eglen SJ, Reese BE. From random to regular: Variation in the patterning of

637 retinal mosaics*. J Comp Neurol. 2020 Sep;528(13):2135-60.

638 34. Amini R, Rocha-Martins M, Norden C. Neuronal Migration and Lamination in the

$639 \quad$ Vertebrate Retina. Front Neurosci. 2017;11:742.

640 35. Breitwieser L, Hesam A, de Montigny J, Vavourakis V, Iosif A, Jennings J, et al.

641 BioDynaMo: a modular platform for high-performance agent-based simulation. Wren J, 642 editor. Bioinformatics. 2021 Sep 16;btab649.

643 36. Cook JE. Spatial properties of retinal mosaics: An empirical evaluation of some existing measures. Vis Neurosci. 1996 Jan;13(1):15-30. 